\title{
Respiratory Pathogen Panel
}

National Cancer Institute

\section{Source}

National Cancer Institute. Respiratory Pathogen Panel. NCI Thesaurus. Code C132852.

A diagnostic test for respiratory pathogens that uses PCR for differential diagnosis during upper or lower respiratory tract infection. 